\title{
Inequalities for Solutions of Mixed Boundary Value Problems for Elastic Plates
}

\author{
J. H. Bramble* and L. E. Payne*
}

(March 4, 1964)

\begin{abstract}
In this paper a number of explicit a priori inequalities for solutions of the plate equation are derived. These inequalities together with the Rayleigh-Ritz technique may be used to compute error bounds in various mixed boundary value problems for elastic plates.
\end{abstract}

\section{Introduction}

In two recent papers $[2,3]^{1}$ the authors presented methods for obtaining pointwise bounds in the three most common boundary value problems for elastic plates. These bounds were of a priori type, that is they held for a class of functions required to satisfy only smoothness conditions. Hence one could approximate the (unknown) solution of one of these problems in terms of essentially arbitrary functions, and the inequalities gave bounds on the error.

In this paper we derive similar a priori bounds in the three most common mixed boundary value problems for elastic plates. For simplicity we consider only the case of a simply connected region $R$ whose boundary $\Sigma$ consists of two disjoint portions $\Sigma_{1}$, and $\Sigma_{2}$ (each connected) on which different sets of boundary conditions are imposed. It will be clear how the results are to be extended if $\Sigma_{1}$, and/or $\Sigma_{2}$ are not connected or if $R$ is multiply connected.

In this paper we shall restrict our attention to the problem of obtaining bounds for the $L_{2}$ integrals of an arbitrary sufficiently smooth function $w$ in terms of $L_{2}$ integrals of quantities which are data whenever the arbitrary function $w$ is actually the solution $u$ to the problem in question. By use of mean value inequalities and the Rayleigh-Ritz technique as indicated in [2,3], the desired pointwise bounds are then obtained. The well known Rayleigh-Ritz technique consists in choosing $w=u-\sum_{i=1}^{N} a_{i} \Phi_{i}$, where the $\Phi_{i}$ are $N$ linearly independent sufficiently smooth functions, and the $a_{i}$ are determined in such a way as to minimize the terms involving the data of $u$.

The particular problems treated here are the following:

Problem I:

$\Delta^{2} u \quad$ prescribed in $R$

$u, \frac{\partial u}{\partial n}$ given on $\Sigma_{1}$

$u, M(u)$ given on $\Sigma_{2}$

Problem II:

$\Delta^{2} u \quad$ prescribed in $R$

$u, \frac{\partial u}{\partial n}$ given on $\Sigma_{1}$

$M(u), V(u)$ given on $\Sigma_{2}$

${ }^{1}$ Figures in brackets indicate the literature references at the end of this paper

*University of Maryland, College Park, Md.: part-time worker at National Bureau of Standards, Washington, D.C. This research was supported in part by the National Science Foundation under Grant NSF-GP-3. 


$$
u, M(u) \text { given on } \Sigma_{1}
$$

$$
M(u), V(u) \text { given on } \Sigma_{2} \text {. }
$$

Here $\Delta^{2}$ is the biharmonic operator $\frac{\partial^{4}}{\partial x^{4}}+2 \frac{\partial^{4}}{\partial x^{2} \partial y^{2}}+\frac{\partial^{4}}{\partial y^{4}}(\Delta$ denotes the Laplace operator), and $\frac{\partial}{\partial n}$ denotes the normal derivative directed outward on $\Sigma$. Also

and

$$
M(u)=\Delta u-(1-\sigma)\left(\frac{\partial^{2} u}{\partial s^{2}}+\frac{1}{\rho} \frac{\partial u}{\partial n}\right)
$$

$$
V(u)=\frac{\partial}{\partial n}(\Delta u)+(1-\sigma)\left[\frac{\partial^{3} u}{\partial s^{2} \partial n}-\frac{\partial}{\partial s}\left(\frac{1}{\rho} \frac{\partial u}{\partial s}\right)\right]
$$

where $\frac{\partial}{\partial s}$ denotes the derivative with respect to arc length on $\Sigma, \sigma$ is Poisson's ratio and $\rho$ is the radius of curvature. The quantities $M(u)$ and $V(u)$ are proportional to the normal moment and the reaction normal to the plate on $\Sigma$, respectively.

In subsequent sections we shall refer to the bounding of integrals of an arbitrary function $w$ in terms of $L_{2}$ integrals of its "data." For example, in problem I the data of $w$ are the quantities $\Delta^{2} w$ in $R, w$ and $\frac{\partial w}{\partial n}$ on $\Sigma_{1}$ and $w$ and $M(w)$ on $\Sigma_{2}$. In the other two problems the "data" of $w$ are defined analogously.

Throughout this paper we assume the boundary $\Sigma$ of $R$ to be sufficiently smooth so that all of the applications of the divergence theorem used are valid. It will be apparent that if the derivatives of the curvature of $\Sigma$ are continuous then there is no question of the validity of the indicated operations provided the functions themselves are smooth enough. It will also be clear that less smoothness of $\Sigma$ can actually be tolerated in each problem, but the determination of the minimum smoothness requirements is not investigated in this paper.

We make use of the summation convention throughout and employ a comma to denote differentiation, e.g., $w,_{i}=\frac{\partial w}{\partial x^{i}}$. We denote the points at which $\Sigma_{1}$ and $\Sigma_{2}$ join as $P_{1}$ and $P_{2}$. Arc length will be measured along $\Sigma$ in the counterclockwise direction.

Additional notation will be defined as the need arises in the text. We turn now to the question of obtaining bounds in problems I, II, and III. These will be discussed separately in sections 2,3 , and 4 . Certain auxiliary inequalities which will be required in treating the three mixed problems will be derived in appendix A.

\section{Problem I}

In a recent paper [4, eq (2.1)] the authors derived the following inequality for a function $w$ with piecewise continuous second derivatives:

$$
\left\{\int_{R} w^{2} d A\right\}^{1 / 2} \leqslant K_{1}\left\{\oint_{\Sigma} w^{2} d S\right\}^{1 / 2}+K_{2}\left\{\int_{R}(\Delta w)^{2} d A\right\}^{1 / 2}
$$

where the constants $K_{1}$ and $K_{2}$ were explicit. (As indicated in [4,5] appropriate constants $K_{1}$ and $K_{2}$ are obtained by making use of results of Payne and Weinberger [10]). Using (2.1) as a starting point we shall derive the desired $L_{2}$ inequality in problem I.

We first define $E(w, w)$ as

$$
E(w, w)=\int_{R}\left[\sigma(\Delta w)^{2}+(1-\sigma) w,{ }_{i j} w, i j\right] d A .
$$


It is well known that $E(u, u)$ is proportional to the strain energy. Since $(\Delta w)^{2} \leqslant 2 w, i j w, i j$ it follows that

$$
\int_{R}(\Delta w)^{2} d A \leqslant \frac{2}{1+\sigma} E(w, w)
$$

Thus inserting (2.3) into (2.1) we obtain

$$
\left\{\int_{R} w^{2} d A\right\}^{1 / 2} \leqslant K_{1}\left\{\oint_{\Sigma} w^{2} d S\right\}^{1 / 2}+\tilde{K}_{2}\{E(w, w)\}^{1 / 2}
$$

where

$$
\tilde{K}_{2}=K_{2}\left[2(1+\sigma)^{-1}\right]^{1 / 2} .
$$

We seek now to bound $E(w, w)$ in terms of the data of problem I. In order to simplify our computations we shall assume that

$$
\lim _{Q \rightarrow P_{1}, P_{2}} \frac{\partial w(Q)}{\partial n}=0, \lim _{Q \rightarrow P_{1}, P_{2}} \frac{\partial^{2} w(Q)}{\partial s \partial n}=0
$$

where $Q$ is a point on $\Sigma_{1}$. This restriction is by no means necessary, since bounds could be derived which involve the values of $\frac{\partial w}{\partial n}$ and $\frac{\partial^{2} w}{\partial s \partial n}$ at $P_{1}$ and $P_{2}$. In applying the a priori type inequalities, however, $w$ will be set equal to $u-\Phi$ where $u$ is the solution to problem I and $\Phi$ is an approximating function. The function $\Phi$ will then be represented as a linear combination of $N$ linearly independent functions $\Phi_{i}$ with arbitrary real coefficients. The restriction (2.5) prescribes four relations among the coefficients. The Rayleigh-Ritz technique together with these four conditions then determine the coefficients. Hence, for practical purposes, (2.5) is no restriction at all. Condition (2.5) permits us to treat the cases in which $\Sigma_{1}$ and $\Sigma_{2}$ do not have end points in common (e.g., certain multiply connected configurations) and the case in which points $P_{1}$ and $P_{2}$ do occur, in a unified manner.

In order to bound $E(w, w)$ in terms of the data of problem I, we decompose $w$ as follows:

$$
w=B+\varphi
$$

where

Clearly then

$$
\begin{gathered}
\Delta^{2} B=0 \text { in } R \\
B=w \text { on } \Sigma \\
\frac{\partial B}{\partial n}=\left\{\begin{array}{c}
\frac{\partial w}{\partial n} \text { on } \Sigma_{1} \\
0 \text { on } \Sigma_{2} .
\end{array}\right.
\end{gathered}
$$

$$
\begin{aligned}
\Delta^{2} \varphi & =\Delta^{2} w \text { in } R \\
\varphi & =0 \text { on } \Sigma \\
\frac{\partial \varphi}{\partial n} & =0 \text { on } \Sigma_{1} \\
M(\varphi) & =M(w)-M(B) \text { on } \Sigma_{2} .
\end{aligned}
$$


We now consider the identity

$$
E(w, w)=E(B, B)+2 E(w, \varphi)-E(\varphi, \varphi)
$$

and note that

$$
E(w, \varphi)=\int_{\Sigma_{2}} \frac{\partial \varphi}{\partial n} M(w) d S+\int_{R} \varphi \Delta^{2} w d A
$$

By Schwarz's inequality we have

$$
|E(w, \varphi)| \leqslant\left\{\int_{\Sigma_{2}}\left(\frac{\partial \varphi}{\partial n}\right)^{2} d S \int_{\Sigma_{2}} M^{2}(w) d S\right\}^{1 / 2}+\left\{\int_{R} \varphi^{2} d A \int_{R}\left(\Delta^{2} w\right)^{2} d A\right\}^{1 / 2} .
$$

Since $\varphi$ vanishes on $\Sigma$, it follows from the duality principle of Fichera [8] that

$$
\left\{\int_{\Sigma}\left(\frac{\partial \varphi}{\partial n}\right)^{2} d S\right\}^{1 / 2} \leqslant K_{1}\left\{\int_{R}(\Delta \varphi)^{2} d A\right\}^{1 / 2}
$$

where $K_{1}$ is the same constant as the $K_{1}$ appearing in (2.1). (In the determination of the explicit value for $K_{1}$ in [5] and [10] the quantity was actually determined for (2.12) rather than (2.1).) It is well known also that if $\varphi$ has piecewise continuous second derivatives in $R$ and vanishes on $\Sigma$ then

$$
\left\{\int_{R} \varphi^{2} d A\right\}^{1 / 2} \leqslant \frac{1}{\lambda_{1}}\left\{\int_{R}(\Delta \varphi)^{2} d A\right\}^{1 / 2}
$$

where $\lambda_{1}$ is the first eigenvalue in the fixed membrane problem for $R$. If this number is not known, lower bounds for it can be easily computed from monotony principles, the Faber-Krahn [7,9] inequality, or other considerations. Inserting (2.12) and (2.13) into (2.11) we obtain

$$
\begin{gathered}
|E(w, \varphi)| \leqslant\left[K_{1}\left\{\int_{\Sigma_{2}}[M(w)]^{2} d S\right\}^{1 / 2}+\frac{1}{\lambda_{1}}\left\{\int_{R}\left(\Delta^{2} w\right)^{2} d A\right\}^{1 / 2}\right]\left\{\int_{R}(\Delta \varphi)^{2} d A\right\}^{1 / 2} \\
\leqslant\left[K_{1}\left\{\int_{\Sigma_{2}}[M(w)]^{2} d S\right\}^{1 / 2}+\frac{1}{\lambda_{1}}\left\{\int_{R}\left(\Delta^{2} w\right)^{2} d A\right\}^{1 / 2}\right]\left[\frac{2}{1+\sigma} E(\varphi, \varphi)\right]^{1 / 2}
\end{gathered}
$$

the last inequality resulting from (2.3). From (2.14) and (2.9) it follows then that

$$
E(w, w) \leqslant E(B, B)+2\left(\frac{2}{1+\sigma}\right)^{1 / 2}\left[K_{1}\left\{\int_{\Sigma_{2}}[M(w)]^{2} d S\right\}^{1 / 2}+\frac{1}{\lambda_{1}}\left\{\int_{R}\left(\Delta^{2} w\right)^{2} d A\right\}^{1 / 2}\right][E(\varphi, \varphi)]^{1 / 2}-E(\varphi, \varphi)
$$

or, using the arithmetic geometric-mean inequality,

$$
E(w, w) \leqslant E(B, B)+\frac{2}{1+\sigma}\left[K_{1}\left\{\int_{\Sigma_{2}}[M(w)]^{2} d S\right\}^{1 / 2}+\frac{1}{\lambda_{1}}\left\{\int_{R}\left(\Delta^{2} w\right)^{2} d A\right\}^{1 / 2}\right]^{2} .
$$

By combining (2.4) and (2.16) we now have a bound for $\int_{R} w^{2} d A$ in terms of $L_{2}$ integrals of the data and $E(B, B)$, where $B$ is the solution to (2.7). We seek now to bound $E(B, B)$ in terms of the data of $w$.

From the divergence theorem we have

$$
\begin{aligned}
E(B, B) & =\int_{R}(\Delta B)^{2} d A+(1-\sigma) \int_{R}\left[B,{ }_{i j} B, i_{i j}-(\Delta B)^{2}\right] d A \\
& =\int_{R}(\Delta B)^{2} d A+(1-\sigma) \oint_{\Sigma} B,{ }_{i}\left(n_{j} \frac{\partial}{\partial x^{i}}-n_{i} \frac{\partial}{\partial x^{j}}\right) B,{ }_{j} d S \\
& =\int_{R}(\Delta B)^{2} d A+(1-\sigma) \oint_{\Sigma}\left\{\frac{\partial B}{\partial s} \frac{\partial^{2} B}{\partial s \partial n}-\frac{\partial B}{\partial n} \frac{\partial^{2} B}{\partial s^{2}}-\frac{1}{\rho}\left[\left(\frac{\partial B}{\partial n}\right)^{2}+\left(\frac{\partial B}{\partial s}\right)^{2}\right]\right\} d S .
\end{aligned}
$$


Now if $\frac{\partial B}{\partial s}$ and $\frac{\partial B}{\partial n}$ are continuous on $\Sigma$ and either $\frac{\partial^{2} B}{\partial s \partial n}\left(\right.$ or $\left.\frac{\partial^{2} B}{\partial s^{2}}\right)$ is square integrable on $\Sigma$, then we

may integrate the first (or second) term in the boundary integral on the right by parts on $\Sigma$ to obtain, for instance,

$$
E(B, B)=\int_{R}(\Delta B)^{2} d A-2(1-\sigma) \int_{\Sigma_{1}} \frac{\partial w}{\partial n} \frac{\partial^{2} w}{\partial s^{2}}-\oint_{\Sigma} \frac{1}{\rho}\left(\frac{\partial w}{\partial s}\right)^{2} d S-\int_{\Sigma_{1}} \frac{1}{\rho}\left(\frac{\partial w}{\partial n}\right)^{2} d S
$$

We have also made use of the boundary conditions (2.7). An application of the arithmetic-geometric mean inequality yields for any positive $\hat{\alpha}$

$E(B, B) \leqslant \int_{R}(\Delta B)^{2} d A+(1-\sigma)^{2} \oint_{\Sigma} \hat{\alpha}\left(\frac{\partial^{2} w}{\partial s^{2}}\right) d S+\int_{\Sigma_{1}}\left(\hat{\alpha}^{-1}-\rho^{-1}\right)\left(\frac{\partial w}{\partial n}\right)^{2} d S-\oint_{\Sigma} \rho^{-1}\left(\frac{\partial w}{\partial s}\right)^{2} d S$.

An inequality bounding the first term on the right of (2.19) in terms of the data of $w$ is derived in appendix A. The insertion of (A.25) into (2.19) together with (2.7) results in the inequality

$E(B, B) \leqslant \hat{d}_{1} \oint_{\Sigma} w^{2} d S+\hat{d}_{2} \oint_{\Sigma}\left(\frac{\partial w}{\partial s}\right)^{2} d S+\hat{d}_{3} \int_{\Sigma_{1}}\left(\frac{\partial w}{\partial n}\right)^{2} d S+\hat{d}_{4} \oint_{\Sigma}\left(\frac{\partial^{2} w}{\partial s^{2}}\right)^{2} d S+\hat{d}_{5} \int_{\Sigma_{1}}\left(\frac{\partial^{2} w}{\partial s \partial n}\right)^{2} d S$

with explicit constants $\hat{d}_{i}$. If the region $R$ is simply connected we can actually obtain the inequality (see appendix A, in particular (A.26)-(A.28))

$$
E(B, B) \leqslant \hat{d}_{6} \oint_{\Sigma} \frac{\partial B,_{i}}{\partial s} \frac{\partial B,_{i}}{\partial s} d S .
$$

We now insert (2.20) and (2.16) back into (2.4) to obtain the desired inequality, which may be written in the form

$$
\begin{aligned}
\int_{R} w^{2} d A \leqslant A_{1} \oint_{\Sigma} w^{2} d S & +A_{2} \oint_{\Sigma}\left(\frac{\partial w}{\partial s}\right)^{2} d S+A_{3} \oint_{\Sigma}\left(\frac{\partial^{2} w}{\partial s^{2}}\right)^{2} d S \\
& +A_{4} \int_{\Sigma_{1}}\left(\frac{\partial w}{\partial n}\right)^{2} d S+A_{5} \int_{\Sigma_{1}}\left(\frac{\partial^{2} w}{\partial s \partial n}\right)^{2} d S+A_{6} \int_{\Sigma_{2}}[M(w)]^{2} d S+A_{7} \int_{R}\left(\Delta^{2} w\right)^{2} d A .
\end{aligned}
$$

Here again the constants $A_{i}$ may be explicitly computed.

In many of our intermediate inequalities sharper results could have been obtained by leaving certain positive weight functions under the integral signs rather than factoring them out.

It is now possible to employ the technique used in [2] and [3] to obtain pointwise bounds in $R$ for $w$ and its derivatives in terms of $L_{2}$ integrals of the data of problem I. It appears likely that by using the results of Bramble and Hubbard [1] it would be possible, if sufficient smoothness of the boundary and data is assumed, to obtain an inequality of the form (2.22) in which $A_{2}, A_{3}$, and $A_{5}$ are zero. However, the remaining constants would then be more complicated functions of the geometry of $\Sigma_{1}$ and $\Sigma_{2}$.

\section{Problem II}

We assume in this section that $R$ and the portion $\Sigma_{2}$ of $\Sigma$ are such that we can define a vector field $f^{i}$ which has piecewise continuous first derivatives in $R$ and satisfies

$$
\begin{aligned}
& f,{ }_{i}^{i}-f^{i} f^{i}>p>0 \text { in } R \\
& -f^{i} n_{i}>q>0 \text { on } \Sigma_{2} .
\end{aligned}
$$


This is of course always possible if $\Sigma$ is sufficiently smooth and $\Sigma_{1}$ is nonempty. In fact, if we take any sufficiently smooth function $\varphi$ which is superharmonic in $R$ and whose normal derivative is positive on $\Sigma_{2}$ then one $\operatorname{such} f^{i}$ is given by

$$
f^{i}=\frac{-\varphi, i}{\varphi+a}
$$

where $a$ is chosen so large that $\varphi+a>0$ in $R$.

In this section we derive a bound for the $L_{2}$ integral of an arbitrary $C^{3}$ function $w$ in terms of $L_{2}$ integrals of the data of problem II. Again in the application of our inequalities we shall set $w=u-\Phi$, where $u$ is the solution to problem II and $\Phi$ is the approximating function. In this section we assume that $\Phi$ has been so chosen that

$$
\lim _{Q \rightarrow P_{1}, P_{2}} w(Q)=\lim _{Q \rightarrow P_{1}, P_{2}} \frac{\partial w(Q)}{\partial s}=\lim _{Q \rightarrow P_{1}, P_{2}} \frac{\partial^{2} w(Q)}{\partial s^{2}}=\lim _{Q \rightarrow P_{1}, P_{2}} \frac{\partial w(Q)}{\partial n}=\lim _{Q \rightarrow P_{1}, P_{2}} \frac{\partial^{2} w(Q)}{\partial s \partial n}=0
$$

If we choose

$$
\Phi=\sum_{i=1}^{N} a_{i} \Phi_{i}
$$

where the $\Phi_{i}$ are linearly independent functions, then (3.2) imposes ten conditions on the $a_{i}$. If $\Sigma_{1}$ and $\Sigma_{2}$ have no points in common then conditions (3.2) are unnecessary.

We now consider the following application of the divergence theorem.

$$
\oint_{\Sigma} f^{i} n_{i} w^{2} d S=\int_{R} f_{{ }_{i}}^{i} w^{2} d A+2 \int_{R} f^{i} w w,{ }_{i} d A .
$$

Upon rearranging and applying the arithmetic-geometric mean inequality, we obtain

$$
\int_{R}\left(f_{i}^{i}-f^{i} f^{i}\right) w^{2} d A \leqslant \oint_{\Sigma} f^{i} n_{i} w^{2} d S+\int_{R}|\operatorname{grad} w|^{2} d A .
$$

Using (3.1) we have

$$
p \int_{R} w^{2} d A+q \int_{\Sigma_{2}} w^{2} d S \leqslant \int_{\Sigma_{1}} f^{i} n_{i} w^{2} d S+\int_{R}|\operatorname{grad} w|^{2} d A
$$

Let us now rewrite (3.4) with $w^{2}$ replaced by $|\operatorname{grad} w|^{2}$. It follows then in the same way that

$$
p \int_{R}|\operatorname{grad} w|^{2} d A+q \int_{\Sigma_{2}}|\operatorname{grad} w|^{2} d S \leqslant \int_{\Sigma_{1}} f^{l} n_{i}|\operatorname{grad} w|^{2} d S+\int_{R} w,{ }_{i j} w,{ }_{i j} d A
$$

Combining the two inequalities and dropping the integrals over $\Sigma_{2}$, we obtain the inequality

$$
\int_{R} w^{2} d A \leqslant p^{-1} \int_{\Sigma_{1}} f^{i} n_{i} w^{2} d S+p^{-2} \int_{\Sigma_{1}} f^{i} n_{i}|\operatorname{grad} w|^{2} d S+p^{-2} \int_{R} w,{ }_{i j} w,{ }_{i j} d A .
$$

Clearly for $\sigma \geqslant 0$

$$
\int_{R} w,{ }_{i j} w,{ }_{i j} d A \leqslant(1-\sigma)^{-1} E(w, w)
$$


If $\sigma<0$, the factor $(1-\sigma)^{-1}$ must be replaced by $2(2-\sigma)^{-1}$. We assume, however, in the following that $\sigma \geqslant 0$. Then

$$
\int_{R} w^{2} d A \leqslant p^{-1} \int_{\Sigma_{1}} f^{i} n_{i} w^{2} d S+p^{-2} \int_{\Sigma_{1}} f^{i} n_{i}|\operatorname{grad} w|^{2} d S+\left[p^{2}(1-\sigma)\right]^{-1} E(w, w) .
$$

In order to bound $E(w, w)$ in terms of the desired quantities, we decompose $w$ as follows:

where $B$ satisfies

$$
w=B+\varphi
$$

$$
\begin{aligned}
\Delta^{2} B & =0 \text { in } R \\
B & =\left\{\begin{array}{c}
w \text { on } \Sigma_{1} \\
0 \text { on } \Sigma_{2}
\end{array}\right. \\
\frac{\partial B}{\partial n} & =\left\{\begin{array}{c}
\frac{\partial w}{\partial n} \text { on } \Sigma_{1} \\
0 \text { on } \Sigma_{2}
\end{array}\right.
\end{aligned}
$$

The function $\varphi$ then satisfies

$$
\begin{gathered}
\Delta^{2} \varphi=\Delta^{2} w \text { in } R \\
\varphi=\frac{\partial \varphi}{\partial n}=0 \text { on } \Sigma_{1} \\
M(\varphi)=M(w)-M(B), V(\varphi)=V(w)-V(B) \text { on } \Sigma_{2} .
\end{gathered}
$$

We again make use of the identity

where now

$$
E(w, w)=E(B, B)+2 E(\varphi, w)-E(\varphi, \varphi)
$$

$$
E(\varphi, w)=\int_{\Sigma_{2}} \frac{\partial \varphi}{\partial n} M(w) d S-\int_{\Sigma_{2}} \varphi V(w) d S+\int_{R} \varphi \Delta^{2} w d A
$$

We apply the Schwarz inequality as follows:

$$
\begin{aligned}
|E(\varphi, w)| \leqslant\left\{q \int_{\Sigma_{2}}\left(\frac{\partial \varphi}{\partial n}\right)^{2} d S+p^{2} \int_{R} \varphi^{2} d A+p q \int_{\Sigma_{2}} \varphi^{2} d S\right\}^{1 / 2} & \left\{q^{-1} \int_{\Sigma_{2}}[M(w)]^{2} d S\right. \\
& \left.+p^{-2} \int_{R}\left(\Delta^{2} w\right)^{2} d A+p^{-1} q^{-1} \int_{\Sigma_{2}}(V(w))^{2} d S\right\}^{1 / 2} .
\end{aligned}
$$

Now, in view of (3.6), the first term on the right of (3.16) may be bounded by

$$
q \int_{\Sigma_{2}}\left(\frac{\partial \varphi}{\partial n}\right)^{2} d S+p^{2} \int_{R} \varphi^{2} d A+p q \int_{\Sigma_{2}} \varphi^{2} d s \leqslant p \int_{R}|\operatorname{grad} \varphi|^{2} d A+q \int_{\Sigma_{2}}|\operatorname{grad} \varphi|^{2} d S .
$$

We have also made use of the fact that $\left(\frac{\partial \varphi}{\partial n}\right)^{2} \leqslant|\operatorname{grad} \varphi|^{2}$. But from (3.7) it follows then that

$$
p \int_{R}|\operatorname{grad} \varphi|^{2} d A+q \int_{\Sigma_{2}}|\operatorname{grad} \varphi|^{2} d S \leqslant \int_{R} \varphi,{ }_{i j} \varphi,{ }_{i j} d A=(1-\sigma)^{-1} E(\varphi, \varphi) .
$$

Thus

$$
|E(\varphi, w)| \leqslant\left\{(1-\sigma)^{-1} E(\varphi, \varphi)\right\}^{1 / 2}\left\{q^{-1} \int_{\Sigma_{2}}(M(w))^{2} d S+p^{-2} \int_{R}\left(\Delta^{2} w\right)^{2} d A+p^{-1} q^{-1} \int_{\Sigma_{2}}(V(w))^{2} d S\right\}^{1 / 2} .
$$


The insertion of (3.19) into (3.14), followed by an application of the arithmetic-geometric mean inequality, yields

$E(w, w) \leqslant E(B, B)+(1-\sigma)^{-1}\left\{q^{-1} \int_{\Sigma_{2}}(M(w))^{2} d S+p^{-2} \int_{R}\left(\Delta^{2} w\right)^{2} d A+p^{-1} q^{-1} \int_{\Sigma_{2}}(V(w))^{2} d S\right\}$.

A combination of (3.10) and (3.20) thus gives us a bound for $\int_{R} w^{2} d A$ in terms of the data of $w$ and $E(B, B)$. We seek now a bound for $E(B, B)$ in terms of the data of $w$.

From (2.17), it follows as before that

$$
E(B, B)=\int_{R}(\Delta B)^{2} d A+2(1-\sigma) \int_{\Sigma_{1}} \frac{\partial w}{\partial s} \frac{\partial^{2} w}{\partial s \partial n} d S-\int_{\Sigma_{1}} \frac{1}{\rho}\left[\left(\frac{\partial w}{\partial s}\right)^{2}+\left(\frac{\partial w}{\partial n}\right)^{2}\right] d S .
$$

Using the arithmetic-geometric mean inequalitv we obtain for positive $\alpha_{8}$

$$
E(B, B) \leqslant \int_{R}(\Delta B)^{2} d A+(1-\sigma)^{2} \alpha_{8} \int_{\Sigma_{1}}\left(\frac{\partial^{2} w}{\partial s \partial n}\right)^{2} d S+\int_{\Sigma_{1}}\left(\alpha_{8}^{-1}-\rho^{-1}\right)\left(\frac{\partial w}{\partial s}\right)^{2} d S-\int_{\Sigma_{1}} \rho^{-1}\left(\frac{\partial w}{\partial n}\right)^{2} d S
$$

As in the previous section we require a bound for $\int_{R}(\Delta B)^{2} d A$ in terms of the data of $w$. The desired bound was computed in appendix A and is given by (A.25), i.e.,

$\int_{R}(\Delta B)^{2} d A \leqslant \bar{d}_{1} \oint_{\Sigma} B^{2} d S+\bar{d}_{2} \oint_{\Sigma}\left(\frac{\partial B}{\partial s}\right)^{2} d S+\bar{d}_{3} \oint_{\Sigma}\left(\frac{\partial B}{\partial n}\right)^{2} d S+\bar{d}_{4} \oint_{\Sigma}\left(\frac{\partial^{2} B}{\partial s \partial n}\right)^{2} d S+\bar{d}_{5} \oint_{\Sigma}\left(\frac{\partial^{2} B}{\partial s^{2}}\right)^{2} d S$.

This inequality may be rewritten, using (3.12), as

$$
\int_{R}(\Delta B)^{2} d A \leqslant \bar{d}_{1} \int_{\Sigma_{1}} w^{2} d S+\bar{d}_{2} \int_{\Sigma_{1}}\left(\frac{\partial w}{\partial s}\right)^{2} d S+\bar{d}_{3} \int_{\Sigma_{1}}\left(\frac{\partial w}{\partial n}\right)^{2} d S+\bar{d}_{4} \int_{\Sigma_{1}}\left(\frac{\partial^{2} w}{\partial s \partial n}\right)^{2} d S+\bar{d}_{5} \int_{\Sigma_{1}}\left(\frac{\partial^{2} w}{\partial s^{2}}\right)^{2} d S
$$

A combination of (3.10), (3.20), (3.22), and (3.24) thus leads to the desired inequality, which is of the form

$$
\begin{aligned}
\int_{R} w^{2} d A \leqslant B_{1} \int_{\Sigma_{1}} w^{2} d S+ & B_{2} \int_{\Sigma_{1}}\left(\frac{\partial w}{\partial s}\right)^{2} d S+B_{3} \int_{\Sigma_{1}}\left(\frac{\partial w}{\partial n}\right)^{2} d S \\
& +B_{4} \int_{\Sigma_{1}}\left(\frac{\partial^{2} w}{\partial s^{2}}\right)^{2} d S+B_{5} \int_{\Sigma_{1}}\left(\frac{\partial^{2} w}{\partial s \partial n}\right)^{2} d S+B_{6} \int_{\Sigma_{2}}[M(w)]^{2} d S \\
& +B_{7} \int_{\Sigma_{2}}[V(w)]^{2} d S+B_{8} \int_{R}\left[\Delta^{2} w\right]^{2} d A
\end{aligned}
$$

where the $B_{i}$ 's are explicit constants.

In many cases (3.25) may be simplified by exploiting some of the geometric properties of $\Sigma$. For instance, whenever $\rho$ is positive on $\Sigma_{1}$ some quantities may be dropped from (3.21) or used to cancel other quantities.

Pointwise bounds in this prob $\mathrm{b}^{\top} \mathrm{m}$ follow from the results of [2] and [3].

\section{Problem III}

This problem is the most difficult of the three since for certain geometrical configurations it does not have a unique solution. Suppose, for instance, that the domain $R$ lies in the right half plane and $\Sigma_{1}$ is a portion of the $y$-axis. In this case the plate may rotate as a rigid body about the $y$-axis. The solution will be unique only up to a rigid body rotation, a term of the form $w=\alpha x$. Note, however, that if $\Sigma_{1}$ is not a straight line then this rigid body motion is no longer possible. Because of the inherent difficulty in treating problem 3 we shall derive in this section 
only an $L_{2}$ bound for the energy integral $E(w, w)$ in terms of the data of the problem. It is possible with such a bound, using the techniques of $[2,3]$, to obtain pointwise bounds for any second derivative of $w$ in terms of $L_{2}$ integrals of the data. In order to simplify the problem somewhat, we assume that $w$ vanishes with its first two tangential derivatives at $P_{1}$ and $P_{2}$. This means merely (as pointed out before) that we put certain restrictions on the approximating functions, and it amounts to six conditions on the arbitrary parameters in the application of the Rayleigh-Ritz technique. With this restriction we decompose $w$ as follows:

where $h$ satisfies

$$
w=h+\tilde{w}
$$

Then

$$
\begin{aligned}
\Delta h & =0 \text { in } R \\
h & =\left\{\begin{array}{lll}
w & \text { on } \Sigma_{1} \\
0 & \text { on } \Sigma_{2}
\end{array} .\right.
\end{aligned}
$$

$$
\begin{aligned}
\Delta^{2} \tilde{w} & =\Delta^{2} w \text { in } R \\
\tilde{w} & =0, M(\tilde{w})=M(w)-(1-\sigma)\left[\frac{\partial^{2} w}{\partial s^{2}}+\frac{1}{\rho} \frac{\partial h}{\partial n}\right] \text { on } \Sigma_{1} \\
M(\tilde{w}) & =M(w)-\frac{1-\sigma}{\rho} \frac{\partial h}{\partial n}, V(\tilde{w})=V(w)+(1-\sigma) \frac{\partial^{3} h}{\partial s^{2} \partial n} \text { on } \Sigma_{2} .
\end{aligned}
$$

Making use of the triangle inequality, we obtain from (4.1)

$$
\{E(w, w)\}^{1 / 2} \leqslant\{E(h, h)\}^{1 / 2}+\{E(\tilde{w}, \tilde{w})\}^{1 / 2} .
$$

We compute first a bound for $E(h, h)$.

Since $h$ is harmonic it follows that

$$
E(h, h)=(1-\sigma) \int_{R} h,{ }_{i j} h,{ }_{i j} d A .
$$

But, as in (2.17), we observe that

$$
\begin{aligned}
\int_{R} h,{ }_{i j} h,{ }_{i j} d A=\int_{\Sigma}\left\{\frac{\partial h}{\partial s} \frac{\partial^{2} h}{\partial s \partial n}-\frac{\partial h}{\partial n} \frac{\partial^{2} h}{\partial s^{2}}-\frac{1}{\rho}\left[\left(\frac{\partial h}{\partial s}\right)^{2}\right.\right. & \left.\left.+\left(\frac{\partial h}{\partial n}\right)^{2}\right]\right\} d S \\
& =-2 \int_{\Sigma}\left[\frac{\partial h}{\partial n} \frac{\partial^{2} h}{\partial s^{2}}+\frac{1}{\rho}\left\{\left(\frac{\partial h}{\partial n}\right)^{2}+\left(\frac{\partial h}{\partial s}\right)^{2}\right\}\right] d S .
\end{aligned}
$$

Applying the arithmetic-geometric mean inequality we obtain

$$
\int_{R} h,{ }_{i j} h,{ }_{i j} d A \leqslant \int_{\Sigma} \alpha_{9}\left(\frac{\partial^{2} h}{\partial s^{2}}\right)^{2} d S+\int_{\Sigma}\left(\alpha_{9}^{-1}-\rho^{-1}\right)\left(\frac{\partial h}{\partial n}\right)^{2} d S-\int_{\Sigma} \rho^{-1}\left(\frac{\partial h}{\partial s}\right)^{2} d S .
$$

If the region is convex, we could choose $\alpha_{9}=\max \rho$ and drop the last two terms. If not, we may use the inequality (A.4) given in appendix A, i.e.,

$$
\oint_{\Sigma}\left(\frac{\partial h}{\partial n}\right)^{2} d S \leqslant b_{0} \oint_{\Sigma} h^{2} d S+b_{1} \oint_{\Sigma}\left(\frac{\partial h}{\partial s}\right)^{2} d S=b_{0} \int_{\Sigma_{1}} w^{2} d S+b_{1} \int_{\Sigma_{1}}\left(\frac{\partial w}{\partial s}\right)^{2} d S .
$$

Upon inserting (4.8) and (4.7) into (4.5), and using the boundary conditions on $h$, we arrive at the inequality

$$
E(h, h) \leqslant(1-\sigma)\left\{\bar{b}_{0} \int_{\Sigma_{1}} w^{2} d S+\bar{b}_{1} \int_{\Sigma_{1}}\left(\frac{\partial w}{\partial s}\right)^{2} d S+\bar{b}_{2} \int_{\Sigma_{1}}\left(\frac{\partial^{2} w}{\partial s^{2}}\right)^{2} d S\right\}
$$

where $\bar{b}_{0}, \bar{b}_{1}$ and $\bar{b}_{2}$ are explicit. 
In order to bound $E(\tilde{w}, \tilde{w})$, we decompose $\tilde{w}$ as follows:

where

$$
\tilde{w}=B+\varphi
$$

$$
\begin{aligned}
\Delta^{2} B & =\Delta^{2} \tilde{w} \text { in } R \\
M(B) & =M(\tilde{w}) \text { on } \Sigma \\
V(B) & =\left\{\begin{array}{l}
V(\tilde{w}) \text { on } \Sigma_{2} \\
-V(h)+\tilde{C}_{1}+\tilde{C}_{2} x+\tilde{C}_{3} y \text { on } \Sigma
\end{array}\right.
\end{aligned}
$$

with $\tilde{C}_{1}, \tilde{C}_{2}$, and $\tilde{C}_{3}$ chosen to insure existence of the function $B$. We indicate now how the $\tilde{C}_{i}$ are to be determined.

Let us choose the coordinate axes in such a way that $\int_{\Sigma_{1}} x d s=\int_{\Sigma_{1}} y d s=\int_{\Sigma_{1}} x y d s=0 . \quad$ Then,

in order that (4.11) may have a solution, it is necessary that the following three equilibrium conditions be satisfied:

$$
\begin{aligned}
& \oint_{\Sigma} V(B) d S=\int_{R} \Delta^{2} B d A \\
& \oint_{\Sigma} x V(B) d S-\oint_{\Sigma} n_{x} M(B) d S=\int_{R} x \Delta^{2} B d A \\
& \oint_{\Sigma} y V(B) d S-\oint_{\Sigma} n_{y} M(B) d S=\int_{R} y \Delta^{2} B d A
\end{aligned}
$$

These three conditions are now used to determine $\tilde{C}_{1}, \tilde{C}_{2}$, and $\tilde{C}_{3}$. We note first that since $h$ is harmonic

$$
\begin{aligned}
\int_{\Sigma} V(h) d S & =0 \\
\int_{\Sigma} x V(h) d S-\int_{\Sigma} n_{x} M(h) d S & =0 \\
\int_{\Sigma} y V(h) d S-\int_{\Sigma} n_{y} M(h) d S & =0 .
\end{aligned}
$$

Thus conditions (4.12) are equivalent to

$$
\begin{aligned}
& \tilde{C}_{1} S_{1}=\int_{R} \Delta^{2} w d A-\int_{\Sigma_{2}} V(w) d S \\
& \tilde{C}_{2} I_{1}=\int_{R} x \Delta^{2} w d A+\int_{\Sigma} n_{x} M(w) d S-\int_{\Sigma_{2}} x V(w) d S \\
& \tilde{C}_{3} I_{2}=\int_{R} y \Delta^{2} w d A+\int_{\Sigma} n_{y} M(w) d S-\int_{\Sigma_{2}} y V(w) d S
\end{aligned}
$$

where $S_{1}$ denotes the length of $\Sigma_{1}$ and

$$
I_{1}=\int_{\Sigma_{1}} x^{2} d S, \quad I_{2}=\int_{\Sigma_{1}} y^{2} d S .
$$

Equations (4.14) thus determine the constants $\tilde{C}_{1}, \tilde{C}_{2}$, and $\tilde{C}_{3}$ in terms of the data of problem III.

In determining the bound for $E(\tilde{w}, \tilde{w})$ of (4.4), we shall, according to (4.10), need a bound for $E(B, B)$. We first indicate how $E(B, B)$ is to be bounded in terms of the data of the problem. We present here a method which is applicable only if the domain $R$ is simply connected. The function 
$B$ is of course determined only up to a rigid body motion, which we assume to be fixed by the conditions

$$
\oint_{\Sigma} B d S=\oint_{\Sigma} \frac{\partial B}{\partial x} d S=\oint_{\Sigma} \frac{\partial B}{\partial y} d S=0
$$

From the results of [3, eq (4.18)], if $R$ is simply connected, it follows that

$$
\left(\frac{2 \pi}{L}\right)^{2} \oint_{\Sigma} B^{2} d S+\oint_{\Sigma}\left(\frac{\partial B}{\partial n}\right)^{2} d S \leqslant \frac{2}{D \bar{p}_{2}(1-\sigma)} E(B, B)
$$

where $\bar{p}_{2}$ is defined in [3, eq $\left.(4.31)\right]$.

From the identity

$$
\oint_{\Sigma} x^{i} n_{i} B^{2} d S=2 \int_{R} B^{2} d A+2 \int_{R} x^{i} B B,{ }_{i} d A
$$

we obtain, using the arithmetic-geometric mean inequality

$$
\int_{R} B^{2} d A \leqslant r_{M} \oint_{\Sigma} B^{2} d S+r_{M}^{2} \int_{R} B,{ }_{i} B,{ }_{i} d A
$$

A repetition of the inequality with $B^{2}$ replaced by $B,{ }_{i} B,{ }_{i}$ yields

$$
\int_{R} B^{2} d A \leqslant r_{M} \oint_{\Sigma} B^{2} d S+r_{M}^{3} \oint_{\Sigma}|\operatorname{grad} B|^{2} d S+r_{M}^{4} \int_{R} B,{ }_{i j} B,{ }_{i j} d A
$$

Here $r_{M}$ denotes the maximum value of $r \equiv \sqrt{x^{i} x^{i}}$ on $\Sigma$. We observed in the proof of (4.17) that

$$
\begin{array}{r}
\oint_{\Sigma} B^{2} d S \leqslant\left(\frac{L}{2 \pi}\right)^{2} \oint_{\Sigma}\left(\frac{\partial B}{\partial s}\right)^{2} d S \\
\oint_{\Sigma}|\operatorname{grad} B|^{2} d S \leqslant \frac{1}{\bar{p}_{2}} \int_{R} B,{ }_{i j} B,{ }_{i j} d A .
\end{array}
$$

Thus from (4.20) and (4.21) we have

$$
\int_{R} B^{2} d A \leqslant \nu \int_{R} B,{ }_{i j} B,{ }_{i j} d A \leqslant \frac{\nu}{1-\sigma} E(B, B)
$$

where

$$
\nu=\left\{r_{M}\left(\frac{L}{2 \pi}\right)^{2}+r_{M}^{3}\right\}\left(\bar{p}_{2}\right)^{-1}+r_{M}^{4}
$$

By Green's identity

$$
\begin{aligned}
E(B, B)= & \int_{\Sigma} \frac{\partial B}{\partial n} M(\tilde{w}) d S-\int_{\Sigma_{2}} B V(\tilde{w}) d S-\int_{\Sigma_{1}} B V(B) d S+\int_{R} B \Delta^{2} w d A=\int_{\Sigma} \frac{\partial B}{\partial n} M(w) d S \\
& -\int_{\Sigma_{2}} B V(w) d S-\int_{\Sigma_{1}}\left(\hat{C}_{1}+\hat{C}_{2} x+\hat{C}_{3} y\right) B d S+\int_{R} B \Delta^{2} w d A-\int_{\Sigma} \frac{\partial B}{\partial n} M(h) d S+\int_{\Sigma} B V(h) d S \\
& =\int_{\Sigma} \frac{\partial B}{\partial n} M(w) d S-\int_{\Sigma_{2}} B V(w) d S-\int_{\Sigma_{1}}\left(\hat{C}_{1}+\hat{C}_{2} x+\hat{C}_{3} y\right) B d S+\int_{R} B \Delta^{2} w d A-E(B, h) .
\end{aligned}
$$

It follows then from Schwarz's inequality that

$$
\begin{aligned}
E(B, B) \leqslant\left\{\int_{\Sigma}\left(\frac{\partial B}{\partial n}\right)^{2} d S+\left(\frac{2 \pi}{L}\right)^{2} \int_{\Sigma} B^{2} d S\right\}^{1 / 2}\left\{\int_{\Sigma}(M(w))^{2} d S+\left(\frac{L}{2 \pi}\right)^{2} \int_{\Sigma}(\bar{V}(w))^{2} d S\right\}^{1 / 2} \\
+\left\{\int_{R} B^{2} d A \int_{R}\left(\Delta^{2} w\right)^{2} d A\right\}^{1 / 2}+\{E(B, B)\}^{1 / 2}\{E(h, h)\}^{1 / 2} .
\end{aligned}
$$


Here $\bar{V}(w)$ is defined as

$$
\bar{V}(w)=\left\{\begin{array}{l}
V(w) \text { on } \Sigma_{2} \\
\tilde{C}_{1}+\tilde{C}_{2} x+\tilde{C}_{3} y \text { on } \Sigma_{1}
\end{array}\right.
$$

From the definition of $\tilde{C}_{1}, \tilde{C}_{2}$, and $\tilde{C}_{3}$ we observe that $\bar{V}(w)$ is data. Using (4.17) and (4.22) we obtain then

$$
\begin{aligned}
\{E(B, B)\}^{1 / 2} \leqslant\left\{\frac{1}{\bar{p}_{2}(1-\sigma)}\left[\int_{\Sigma}(M(w))^{2} d S+\left(\frac{L}{2 \pi}\right)^{2} \int_{\Sigma}(\bar{V}(w))^{2} d S\right]\right\}^{1 / 2} & \\
& +\left\{\frac{\nu}{1-\sigma} \int_{R}\left(\Delta^{2} w\right)^{2} d A\right\}^{1 / 2}+\{E(h, h)\}^{1 / 2},
\end{aligned}
$$

where the bound for the last term is given by (4.9). With (4.27) we now compute the bound for $E(\tilde{w}, \tilde{w})$.

From (4.10) it follows that

$$
E(\tilde{w}, \tilde{w})=E(B, B)+2 E(\tilde{w}, \varphi)-E(\varphi, \varphi)
$$

where

$$
\begin{aligned}
E(\tilde{w}, \varphi) & =\int_{\Sigma} \frac{\partial \tilde{w}}{\partial n} M(\varphi) d S-\int_{\Sigma} \tilde{w} V(\varphi) d S+\int_{R} \tilde{w} \Delta^{2} \varphi d A \\
& =0
\end{aligned}
$$

from (4.10) and (4.11). Thus

$$
E(\tilde{w}, \tilde{w}) \leqslant E(B, B)
$$

Combining (4.4), (4.9), and (4.27) we now obtain (after an application of the arithmetic-geometric mean inequality) an inequality of the form

$$
\begin{aligned}
E(w, w) \leqslant D_{1} \int_{\Sigma_{1}} w^{2} d S & +D_{2} \int_{\Sigma_{1}}\left(\frac{\partial w}{\partial s}\right)^{2} d S+D_{3} \int_{\Sigma_{1}}\left(\frac{\partial^{2} w}{\partial s^{2}}\right)^{2} d S \\
& +D_{4} \oint_{\Sigma}(M(w))^{2} d S+D_{5} \oint_{\Sigma}(\bar{V}(w))^{2} d S+D_{6} \int_{R}\left(\Delta^{2} w\right)^{2} d A
\end{aligned}
$$

which is the desired result.

\section{Appendix $\AA$}

In treating the problems discussed in sections 2,3 , and 4 we required bounds for certain integrals of derivatives of biharmonic functions in terms of their Dirichlet data. We derive the necessary inequalities here, the most important of which is (A.25).

Consider the following Dirichlet problem for $R$ :

$$
\begin{gathered}
\Delta^{2} B=0 \text { in } R \\
B, \frac{\partial B}{\partial n} \text { given on } \Sigma .
\end{gathered}
$$

Let us compute a bound for $\int_{R}(\Delta B)^{2} d A$ in terms of the data of $B$. We assume that the data are such that $\partial^{2} B / \partial s^{2}$ and $\partial^{2} B / \partial s \partial n$ are square integrable on $\Sigma$. 
Let $h$ be the harmonic function which is equal to $B$ on the boundary. Then from Green's identity we have

$$
\int_{R}(\Delta B)^{2} d A=\int_{R}[\Delta(B-h)]^{2} d A=\int_{\Sigma} \frac{\partial}{\partial n}(B-h) \Delta B d S .
$$

Using the arithmetic-geometric mean inequality we obtain, for any positive $\alpha_{0}$, the inequality

$$
\begin{aligned}
\int_{R}(\Delta B)^{2} d A \leqslant \frac{\alpha_{0}}{2} \oint_{\Sigma}(\Delta B)^{2} d S+\frac{1}{2 \alpha_{0}} \oint_{\Sigma}\left[\frac{\partial}{\partial n}(B\right. & -h)]^{2} d S \\
& \leqslant \frac{\alpha_{0}}{2} \oint_{\Sigma}(\Delta B)^{2} d S+\frac{1}{\alpha_{0}}\left[\oint_{\Sigma}\left(\frac{\partial B}{\partial n}\right)^{2} d S+\oint_{\Sigma}\left(\frac{\partial h}{\partial n}\right)^{2} d S\right] .
\end{aligned}
$$

In $[10,11]$ Payne and Weinberger have obtained the following inequality for the last integral in (A.3):

$$
\oint_{\Sigma}\left(\frac{\partial h}{\partial n}\right)^{2} d S \leqslant b_{0} \oint_{\Sigma} h^{2} d S+b_{1} \oint_{\Sigma}\left(\frac{\partial h}{\partial s}\right)^{2} d s=b_{0} \oint_{\Sigma} B^{2} d s+b_{1} \oint_{\Sigma}\left(\frac{\partial B}{\partial s}\right)^{2} d S
$$

The constants $b_{0}$ and $b_{1}$ are explicitly determined. We seek now a bound for the first integral on the right of (A.3).

For an arbitrary sufficiently smooth vector field $g^{i}$ and an arbitrary harmonic function $H$ we consider the following identity:

$\mathbf{0}=\int_{R} g^{k}(B-H),{ }_{k} \Delta^{2} B d A=\oint_{\Sigma} g^{k}(B-H),{ }_{k} \frac{\partial}{\partial n}(\Delta B) d S$

$$
-\int_{R} g,{ }_{l}^{k}(B-H),{ }_{k} \Delta B,{ }_{l} d s-\int_{R} g^{k}(B-H),{ }_{k l} \Delta B,{ }_{l} d A .
$$

Integrating by parts further we obtain

$$
\begin{aligned}
\int_{\Sigma} g^{i} \Delta B\left[n_{k} \frac{\partial}{\partial x^{i}}\right. & \left.-n_{i} \frac{\partial}{\partial x^{k}}\right](B-H),{ }_{k} d S+\frac{1}{2} \oint_{\Sigma} g^{k} n_{k}(\Delta B)^{2} d S+\int_{\Sigma} g^{k}(B-H),{ }_{k} \frac{\partial(\Delta B)}{\partial n} d S \\
& +\int_{\Sigma} g,{ }_{k}^{i} n_{k}(B-H),{ }_{i} \Delta B d S=\int_{R}\left\{\Delta g^{i}(B-H),{ }_{i}+2 g,{ }_{k}^{i}(B-H),{ }_{i k}-\frac{1}{2} g,{ }_{i}^{i} \Delta B\right\} \Delta B d A .
\end{aligned}
$$

Suppose now that a vector function $g^{i}$ with bounded second derivatives has been found such that

$$
g^{i}=n_{i}
$$

on $\Sigma$. It $\Sigma \epsilon C^{2}$ one possible vector field $g^{i}$ is given in appendix B. With $g^{i}$ so chosen we take $H$ to satisfy

$$
\begin{gathered}
\Delta H=0 \text { in } R \\
\frac{\partial H}{\partial n}=\frac{\partial B}{\partial n}-S^{-1} \oint_{\Sigma} \frac{\partial B}{\partial n} d S
\end{gathered}
$$

where $S$ denotes the length of $\Sigma$. From (A.7) and (A.8) it follows that the third boundary integral on the left of (A.6) vanishes (since $B$ is biharmonic).

Since $g^{i}=n_{i}$ on $\Sigma$, the first term in (A.6) becomes

$$
\oint_{\Sigma} n_{i} \Delta B\left(n_{k} \frac{\partial}{\partial x^{i}}-n_{i} \frac{\partial}{\partial x^{k}}\right)(B-H),{ }_{k} d S=\oint_{\Sigma} \Delta B\left[-\frac{\partial^{2}}{\partial s^{2}}-\frac{1}{\rho} \frac{\partial}{\partial n}\right](B-H) d S
$$

while the fourth term on the left may be written as

$$
\oint_{\Sigma} n_{i, k} n_{k}(B-H),{ }_{i} \Delta B d S=\oint_{\Sigma} \frac{\partial g^{i}}{\partial n}(B-H),{ }_{i} \Delta B d S .
$$


Inserting (A.9) and (A.10) into (A.6), and making use of (A.7) and (A.8), we obtain

$$
\begin{aligned}
\frac{1}{2} \oint_{\Sigma}(\Delta B)^{2} d S & =\oint_{\Sigma} \Delta B \frac{\partial^{2}}{\partial s^{2}}(B-H) d S+S^{-1} \oint_{\Sigma}(\Delta B) \rho^{-1} d S \oint_{\Sigma} \frac{\partial B}{\partial n} d S-\oint_{\Sigma} \frac{\partial g^{i}}{\partial n}\left\{\frac{\partial}{\partial s}(B-H) \frac{\partial x^{i}}{\partial s}\right. \\
& \left.+n_{i} S^{-1} \oint_{\Sigma} \frac{\partial B}{\partial n} d S\right\} \Delta B d S+\int_{R} \Delta B\left\{\Delta g^{i}(B-H),{ }_{i}+2 g_{,}^{i}{ }_{k}(B-H),{ }_{i k}-\frac{1}{2} g,{ }_{i}^{i} \Delta B\right\} d A .
\end{aligned}
$$

We assume now that $g^{i}$ and its second derivatives are uniformly bounded in $R$. Then by making use of the arithmetic-geometric mean inequality we obtain for arbitrary positive constants $\alpha_{i}$

$$
\begin{aligned}
&\left(1-\alpha_{1}-\alpha_{2}-\alpha_{3}-\alpha_{4}\right) \oint_{\Sigma}(\Delta B)^{2} d S \leqslant \frac{1}{\alpha_{1}} \oint_{\Sigma}\left[\frac{\partial^{2}}{\partial s^{2}}(B-H)\right]^{2} d S+\frac{\left(p^{-2}\right)_{\max }}{\alpha_{2}} \oint_{\Sigma}\left(\frac{\partial B}{\partial n}\right)^{2} d S \\
&+\frac{C_{1}}{\alpha_{3}} \oint_{\Sigma}\left[\frac{\partial}{\partial s}(B-H)\right]^{2} d S+\frac{C_{2}}{\alpha_{4}} \oint_{\Sigma}\left(\frac{\partial B}{\partial n}\right)^{2} d S+\left(\alpha_{5}+\alpha_{6}+C_{3}\right) \int_{R}(\Delta B)^{2} d A \\
&+\frac{C_{4}}{\alpha_{5}} \int_{R}(B-H),,_{i}(B-H),{ }_{i} d A+\frac{C_{5}}{\alpha_{6}} \int_{R}(B-H),{ }_{i j}(B-H),{ }_{i j} d A .
\end{aligned}
$$

In (A.12) we have used the abbreviations

$$
\begin{aligned}
& C_{1}=\max _{\Sigma}\left[\frac{\partial g^{i}}{\partial n} \frac{\partial x^{i}}{\partial s}\right]^{2} \\
& C_{2}=\max _{\Sigma}\left[\frac{\partial g^{i}}{\partial n} n_{i}\right]^{2} \\
& C_{3}=\max _{R}\left(g,{ }_{i}^{i}\right)^{2} \\
& C_{4}=\max _{R}\left[\Delta g^{i} \Delta g^{i}\right]^{2} \\
& C_{5}=4 \max _{R}\left|g,{ }_{k}^{i} g,{ }_{k}^{i}\right| .
\end{aligned}
$$

We need now bounds for $\int_{R}(B-H),{ }_{i}(B-H),{ }_{i} d A, \int_{R}(B-H),{ }_{i j}(B-H),{ }_{i j} d A$ and $L_{2}$ integrals over $\Sigma$ of the first and second tangential derivatives of $H$, in terms of the data of $B$. We consider first $\int_{R}(B-H),{ }_{i j}(B-H),{ }_{i j} d A$. An integration by parts gives (see 2.17)

$$
\begin{aligned}
\int_{R}(B-H),{ }_{i j}(B-H),{ }_{i j} d A=2 \oint_{\Sigma} \frac{\partial^{2}(B-H)}{\partial s \partial n} \frac{\partial(B-H)}{\partial s} & d S \\
& \quad+\int_{R}(\Delta B)^{2} d A-\oint_{\Sigma} \frac{1}{\rho}\left[(B-H),{ }_{i}(B-H),{ }_{i}\right] d S .
\end{aligned}
$$

The first term on the right vanishes in view of (A.8). Thus

$$
\begin{aligned}
& \int_{R}(B-H),{ }_{i j}(B-H),{ }_{i j} d A=\int_{R}(\Delta B)^{2} d A-S^{-2} \oint_{\Sigma} \frac{1}{\rho} d S\left(\oint_{\Sigma} \frac{\partial B}{\partial n} d s\right)^{2}-\oint_{\Sigma} \frac{1}{\rho}\left[\frac{\partial}{\partial s}(B-H)\right]^{2} d S \\
& \leqslant \int_{R}(\Delta B)^{2} d A+C_{6} \oint_{\Sigma}\left(\frac{\partial B}{\partial n}\right)^{2} d S+C_{6} \oint_{\Sigma}\left[\frac{\partial}{\partial s}(B-H)\right]^{2} d S
\end{aligned}
$$

where $C_{6}$ is given by

$$
C_{6}=\left\{\begin{array}{l}
0 \text { if } \rho \geqslant 0 \text { on } \Sigma \\
\left(-\frac{1}{\rho}\right)_{\max } \text { if } \rho \text { changes sign on } \Sigma .
\end{array}\right.
$$


In a recent paper [6] the authors obtained the following inequality:

$$
\begin{aligned}
\int_{R}(B-H),{ }_{i}(B-H),{ }_{i} d A \leqslant C_{7} \oint_{\Sigma}\left[\frac{\partial}{\partial n}(B-H)\right]^{2} d S+C_{8} \int_{R}(\Delta B)^{2} d A & \\
& \leqslant C_{7} \oint_{\Sigma}\left(\frac{\partial B}{\partial n}\right)^{2} d S+C_{8} \int_{R}(\Delta B)^{2} d A
\end{aligned}
$$

with explicit constants $C_{7}$ and $C_{8}$. The second inequality results from insertion of the boundary condition in (A.8) followed by an application of Schwarz's inequality. The insertion of (A.15) and (A.17) into (A.12) then gives

$$
\begin{aligned}
&\left(1-\alpha_{1}-\alpha_{2}-\alpha_{3}-\alpha_{4}\right) \oint_{\Sigma}(\Delta B)^{2} d S \leqslant \frac{1}{\alpha_{1}} \oint_{\Sigma}\left[\frac{\partial^{2}}{\partial s^{2}}(B-H)\right]^{2} d S \\
&+\left[\frac{\left(\rho^{-2}\right)_{\max }}{\alpha_{2}}+\frac{C_{2}}{\alpha_{4}}+\frac{C_{5} C_{6}}{\alpha_{6}}+\frac{C_{4} C_{7}}{\alpha_{5}}\right] \oint_{\Sigma}\left(\frac{\partial B}{\partial n}\right)^{2} d S+\left[\frac{C_{1}}{\alpha_{3}}+\frac{C_{5} C_{6}}{\alpha_{6}}\right] \oint_{\Sigma}\left[\frac{\partial}{\partial s}(B-H)\right]^{2} d S \\
&+\left[\alpha_{5}+\alpha_{6}+C_{3}+\frac{C_{5}}{\alpha_{6}}+\frac{C_{4} C_{8}}{\alpha_{5}}\right] \int_{R}(\Delta B)^{2} d A .
\end{aligned}
$$

We now choose the constants $\alpha_{i}$ in such a way that

$$
\gamma \equiv 1-\alpha_{1}-\alpha_{2}-\alpha_{3}-\alpha_{4}>0 .
$$

For simplicity we write (A.18) as

$$
\begin{aligned}
\gamma_{1} \oint_{\Sigma}(\Delta B)^{2} d S \leqslant \gamma_{2} \oint_{\Sigma}\left[\frac{\partial^{2}}{\partial s^{2}}(B-H)\right]^{2} d S+\gamma_{3} \oint_{\Sigma}\left(\frac{\partial B}{\partial n}\right)^{2} d S \\
+\gamma_{4} \oint_{\Sigma}\left[\frac{\partial}{\partial s}(B-H)\right]^{2} d S+\gamma_{5} \int_{R}(\Delta B)^{2} d A .
\end{aligned}
$$

We now insert (A.4) and (A.20) back into (A.3) to obtain

$$
\begin{aligned}
\left(\gamma_{1}-\frac{\alpha_{0} \gamma_{5}}{2}\right) \int_{R}(\Delta B)^{2} d A \leqslant \gamma_{2} & \oint_{\Sigma}\left[\frac{\partial^{2}}{\partial s^{2}}(B-H)\right]^{2} d S+\left(\gamma_{3}+\frac{\gamma_{1}}{\alpha_{0}}\right) \oint_{\Sigma}\left(\frac{\partial B}{\partial n}\right)^{2} d S \\
& +\gamma_{4} \oint_{\Sigma}\left[\frac{\partial}{\partial s}(B-H)\right]^{2} d S+\frac{\gamma_{1} b_{0}}{\alpha_{0}} \oint_{\Sigma} B^{2} d S+\frac{\gamma_{1} b_{1}}{\alpha_{6}} \oint_{\Sigma}\left(\frac{\partial B}{\partial s}\right)^{2} d S .
\end{aligned}
$$

The constant $\alpha_{0}$ may be taken as $\gamma_{1} / \gamma_{5}$ which yields the inequality:

$$
\begin{aligned}
\frac{\gamma_{1}}{2} \int_{R}(\Delta B)^{2} d A \leqslant \gamma_{2} \oint_{\Sigma}\left[\frac{\partial^{2}}{\partial s^{2}}(B-H)\right]^{2} d S+\left(\gamma_{3}+\gamma_{5}\right) \oint_{\Sigma}\left(\frac{\partial B}{\partial n}\right)^{2} d S \\
+\gamma_{4} \oint_{\Sigma}\left[\frac{\partial}{\partial s}(B-H)\right]^{2} d S+b_{0} \gamma_{5} \oint_{\Sigma} B^{2} d S+b_{1} \gamma_{5} \oint_{\Sigma}\left(\frac{\partial B}{\partial s}\right)^{2} d S .
\end{aligned}
$$

We need now to bound the tangential derivatives of $H$ in terms of its normal derivatives. From the results of $[10,11]$ we have

$$
\oint_{\Sigma}\left(\frac{\partial H}{\partial s}\right)^{2} d S \leqslant \tilde{C}_{9} \oint_{\Sigma}\left(\frac{\partial H}{\partial n}\right)^{2} d S+\bar{C}_{9} \int_{R} H,{ }_{i} H,{ }_{i} d A \leqslant C_{9} \int_{\Sigma}\left(\frac{\partial B}{\partial n}\right)^{2} d S
$$

with explicit constants $\tilde{C}_{9}, \bar{C}_{9}$, and $C_{9}$. An inequality of type (A.17) has been used to obtain (A.23).

We require finally a bound for $\oint_{\Sigma} \frac{\partial^{2} H}{\partial s^{2}} d S$. 
Since $H$ is harmonic we obtain, on applying an inequality of Bramble and Hubbard [1],

$\oint_{\Sigma}\left[\frac{\partial^{2} H}{\partial s^{2}}\right]^{2} d S \leqslant \tilde{C}_{10} \oint_{\Sigma}\left[\frac{\partial^{2} H}{\partial s \partial n}\right]^{2} d S+\tilde{C}_{11} \oint_{\Sigma}\left(\frac{\partial H}{\partial n}\right)^{2} d S \leqslant C_{10} \oint_{\Sigma}\left[\frac{\partial^{2} B}{\partial s \partial n}\right]^{2} d S+C_{11} \oint_{\Sigma}\left(\frac{\partial B}{\partial n}\right)^{2} d S$

with explicit constants $C_{10}$ and $C_{11}$ (see Appendix C). A use of the arithmetic-geometric mean inequality in the first and third terms on the right of (A.22), together with (A.23) and (A.24), yields the inequality

$$
\begin{aligned}
& \frac{\gamma_{1}}{2} \int_{R}(\Delta B)^{2} d A \leqslant 2 \gamma_{2} \oint_{\Sigma}\left(\frac{\partial^{2} B}{\partial s^{2}}\right)^{2} d S+2 \gamma_{2} C_{10} \oint_{\Sigma}\left(\frac{\partial^{2} B}{\partial s \partial n}\right)^{2} d S+\left(2 \gamma_{4}+b, \gamma_{5}\right) \oint_{\Sigma}\left(\frac{\partial B}{\partial s}\right)^{2} d S \\
& +\left(\gamma_{3}+\gamma_{5}+2 \gamma_{2} C_{11}+2 \gamma_{4} C_{9}\right) \oint_{\Sigma}\left(\frac{\partial B}{\partial n}\right)^{2} d S+b_{0} \gamma_{5} \oint_{\Sigma} B^{2} d S \\
& \leqslant d_{1} \oint_{\Sigma} B^{2} d S+d_{2} \oint_{\Sigma}\left(\frac{\partial B}{\partial s}\right)^{2} d S+d_{3} \oint_{\Sigma}\left(\frac{\partial B}{\partial n}\right)^{2} d S+d_{4} \oint_{\Sigma}\left[\frac{\partial B, i}{\partial s} \frac{\partial B, i}{\partial s}\right] d S
\end{aligned}
$$

which was to be derived.

It might be noted that the left hand side is unaffected by considering $B$ instead of $B$, where

$$
\tilde{B}=B+a_{0}+a_{1} x+a_{2} y \text {. }
$$

In (A.26) the constants $a_{i}$ are determined by the conditions that

$$
\oint_{\Sigma} \tilde{B} d S=\oint_{\Sigma} \tilde{B},{ }_{x} d S=\oint_{\Sigma} \tilde{B},{ }_{y} d S=0
$$

Then, as indicated in [3], it is possible, if $R$ is simply connected, to replace (A.25) by

$$
\int_{R}(\Delta B)^{2} d A \leqslant d_{5} \oint_{\Sigma} \frac{\partial \tilde{B},,_{i}}{\partial s} \frac{\partial \tilde{B},_{i}}{\partial s} d S=d_{5} \oint_{\Sigma} \frac{\partial B, i}{\partial s} \frac{\partial B,_{i}}{\partial s} d S .
$$

\section{Appendix B. The Vector Field $g^{i}$}

We make the assumption that $\Sigma \epsilon C^{3}$ and consider the family of parallel surfaces to $\Sigma$ in $R$ given by $N(x)=$ constant, with $\Sigma$ defined by $N(x)=0$. The parallel surfaces need only be defined in the neighborhood of $\Sigma$ and we shall be interested in the subdomain of $R$ given by $0 \leqslant N(x) \leqslant K^{-1}$ where $K$ is a bound on the curvature of $\Sigma$. The outward normal vector $n_{i}$ on the parallel surface is given by

$$
n_{i}=\frac{-N,{ }_{i}}{\left\{N,{ }_{j} N,{ }_{j}\right\}^{1 / 2}}
$$

It is clear then that if we define $g^{i}$ as

$$
g^{i}=\left\{\begin{array}{l}
(1-K N)^{2} n_{i}, 0 \leqslant N \leqslant K^{-1} \\
0 \text { otherwise }
\end{array}\right.
$$

then $g^{i}=n_{1}$ on $\Sigma$ and $g^{i} \epsilon C^{2}$ in $R$.

\section{Appendix C}

The inequality (A.24) follows from eq (2.6) of [1] by making use of [1, eq (2.2)] to prescribe the arbitrary vectors $g^{i}$ and symmetric tensors $A^{i j}$. We sketch here briefly how the argument goes.

For sufficiently smooth $g^{i}$ and $A^{i j}$ we have the following identity for any harmonic function $H$ :

$$
\int_{R}\left[2\left(g^{m} A^{i j}\right),{ }_{l} H,{ }_{j m}-\left(g^{m} A^{i j}\right),{ }_{m} H,{ }_{j l}\right] H,{ }_{i l} d A=\oint_{\Sigma} g^{m} A^{i j} H,{ }_{i l}\left(2 H,{ }_{j m} n_{l}-H,{ }_{j l} n_{m}\right) d S .
$$


Suppose $g^{i}$ and $A^{i j}$ to be so chosen that on $\Sigma$

$$
\begin{aligned}
g^{i} & =n_{i} \\
A^{i j} & =\delta_{i j}-n_{i} n_{j}
\end{aligned}
$$

If $\Sigma$ is sufficiently smooth, we may choose $g^{i}$ as in appendix B and

$$
A^{i j}=\delta_{i j}-g^{i} g^{j}
$$

We now decompose the derivatives on the boundary into normal and tangential derivatives to obtain

$$
\begin{aligned}
& \oint_{\Sigma}\left[n_{m}\left\{\delta_{i j}-n_{i} n_{j}\right\} H,{ }_{i l}\left(2 H,{ }_{j m} n_{l}-H,{ }_{j l} n_{m}\right)\right] d S \\
&=\oint_{\Sigma}\left\{\left(\frac{\partial^{2} H}{\partial s \partial n}\right)^{2}-\left(\frac{\partial^{2} H}{\partial s^{2}}\right)^{2}-\frac{2}{\rho} \frac{\partial H}{\partial s} \frac{\partial^{2} H}{\partial s \partial n}+\frac{1}{\rho^{2}}\left(\frac{\partial H}{\partial s}\right)^{2}\right. \\
&\left.-\left(\frac{\partial n_{1}}{\partial s} \frac{\partial H}{\partial s}-\frac{\partial n_{2}}{\partial n} \frac{\partial H}{\partial n}\right)^{2}-\left(\frac{\partial n_{2}}{\partial s} \frac{\partial H}{\partial s}+\frac{\partial n_{1}}{\partial n} \frac{\partial H}{\partial n}\right)^{2}\right\} d S .
\end{aligned}
$$

Inserting (C.3) back into (C.1), we have upon rearranging and dropping the last two terms

$$
\oint_{\Sigma}\left(\frac{\partial^{2} H}{\partial s^{2}}\right)^{2} d S \leqslant \oint_{\Sigma}\left\{\left(\frac{\partial^{2} H}{\partial s \partial n}\right)^{2}-\frac{2}{\rho} \frac{\partial H}{\partial s} \frac{\partial^{2} H}{\partial s \partial n}+\frac{1}{\rho^{2}}\left(\frac{\partial H}{\partial s}\right)^{2}\right\} d S+\bar{k} \int_{R} H,{ }_{i j} H,{ }_{i j} d A,
$$

where $\bar{k}$ is easily determined once $g^{i}$ and $A^{i j}$ are given. From (A.8) it follows that

$$
\oint_{\Sigma}\left(\frac{\partial^{2} H}{\partial s^{2}}\right)^{2} d S \leqslant \oint_{\Sigma}\left\{\left(\frac{\partial^{2} B}{\partial s \partial n}\right)^{2}-\frac{2}{\rho} \frac{\partial H}{\partial s} \frac{\partial^{2} B}{\partial s \partial n}+\frac{1}{\rho^{2}}\left(\frac{\partial H}{\partial s}\right)^{2}\right\} d S+\bar{k} \int_{R} H,{ }_{i j} H,{ }_{i j} d A .
$$

But from (4.16)

$$
\int_{R} H,{ }_{i j} H,{ }_{i j} d A=2 \oint_{\Sigma} \frac{\partial^{2} B}{\partial s \partial n} \frac{\partial H}{\partial s} d S-\oint_{\Sigma} \frac{1}{\rho}\left[\left(\frac{\partial H}{\partial n}\right)^{2}+\left(\frac{\partial H}{\partial s}\right)^{2}\right] d S
$$

If we now use the arithmetic-geometric mean inequality in the second term on the right of (C.5) and the second term on the right of (C.6), we obtain an inequality of the form

$$
\oint_{\Sigma}\left(\frac{\partial^{2} H}{\partial s^{2}}\right)^{2} d S \leqslant \bar{K}_{1} \oint_{\Sigma}\left(\frac{\partial^{2} B}{\partial s \partial n}\right)^{2} d S+\bar{K}_{2} \oint_{\Sigma}\left(\frac{\partial H}{\partial s}\right)^{2} d S+\bar{K}_{3} \oint_{\Sigma}\left(\frac{\partial H}{\partial n}\right)^{2} d S .
$$

We now use (A.23) and apply Schwarz's inequality to the last term on the right after insertion of the boundary condition. This leads to (A.24).

\section{References}

[1] Bramble, J. H. and Hubbard, B. E., Some higher order integral identities with applications to bounding techniques, J. Res. NBS 65B, No. 4, 269-276 (1961).

[2] Bramble, J. H. and Payne, L. E., Pointwise bounds in the first biharmonic boundary value problem, J. Math. Phys. 42, 278-286 (1963).

[3] Bramble, J. H. and Payne, L. E., Error bounds in the pointwise approximation of solutions of elastic plate problems. J. Res. NBS 67B, No. 3, 145-155 (1963).

[4] Bramble, J. H. and Payne, L. E., Bounds for derivatives in the Dirichlet problem for Poisson's equation, J. Soc. Ind. Appl. Math. 10, pp. 370-380 (1962).

[5] Bramble, J. H. and Payne, L. E., Bounds for solutions of second order elliptic partial differential equations. Contrib. Diff. Eq. 1, pp. 95-127 (1963). 
[6] Bramble, J. H. and Payne, L. E., Bounds in the Neumann Problem for second order uniformly elliptic operators, Pacific J. Math. 12, 823-833 (1962).

[7] Faber, G., Beweis dass unter aller homogener Membranen von gleicher Fläche und gleìcher Spannung die kreisformige den tiefsten Grundton gibt, Bayr. Akad. Wiss. Sitz., pp. 169-172 (1923).

[8] Fichera, G., Siu un principio di dualità per talune formole di maggiorazione relative alle equazioni differenziali, Atti Accad. Naz. Lincei 19, pp. 411-418 (1955); see also Methods of linear functional analysis in mathematical physics, Proc. Inter. Cong. Math. Amsterdam Vol. 3, pp. 216-228 (1954).

[9] Krahn, E. Über eine von Rayleigh formulierte Minimaleigenschaft des Kreises, Math. Ann. 94, pp. 97-100 (1924); see also, Über Minimaleigenschaften der Kugel in drei und mehr Dimensionen, Acta Comm. Univ. Dorp. A9, pp. 1-44 (1926).

[10] Payne, L. E. and Weinberger, H. F., New bounds in harmonic and biharmonic problems, J. Math. Phys. 4, pp. 291-307 (1955).

[11] Payne, L. E. and Weinberger, H. F., New bounds for solutions of second order partial differential equations, Pac. J. Math. Vol. 8, pp. 551-573 (1958).

(Paper 68B2-121) 